\title{
Solving the rod-and-frame test in a tachistoscopic presentation: Effects of stimulus size and perceptual style
}

\author{
CHRISTIAN MARENDAZ, FRÉDÉRIQUE BRENET, and THÉOPHILE OHLMANN \\ Université des Sciences Sociales de Grenoble, Grenoble, France \\ and \\ CHRISTIAN RAPHEL \\ Centre de Recherches des Services de Santé de l'Armée de Grenoble, Grenoble, France
}

\begin{abstract}
This research was undertaken to demonstrate, with correlational evidence, that presenting the rod-and-frame test (RFT) with either limited (tachistoscopic) or unlimited (Oltman's, 1968, portable RFT) exposure time does not significantly affect the ranking of subjects. The underlying hypothesis is that the intersubject variability of performance on the portable RFT is due essentially to differences in sensitivity to the optostatic vection that appears automatically and almost immediately. Results of the tachistoscopic test show that the effect of angular size of the stimulus is similar to that described in the literature for unlimited time situations, and that subjects' ranking is very similar regardless of the exposure time $(W=.80)$. However, although a difference is observed between the means of low and high achievers in both types of RFT, intraindividual intertask homogeneity (correlation coefficient) is not verified in each of these subgroups. From these results, one can distinguish two processes in the RFT: the first, vections, has to do with the subject's postural orientation and seems to play a great role in organizing interindividual differences. The second process, a more cognitive one, has a less striking effect and has to do with the selection of reference frames in perceptual organization.
\end{abstract}

The vertical adjustment of a rod inside a tilted frame (rod-and-frame test, or RFT) produces deviations in the same direction as the orientation of the frame (Witkin \& Asch, 1948). A large rotating or oscillating disk (Dichgans, Young, Brandt, \& Held, 1972; Hughes, Brecher, \& Fishkin, 1972; Mauritz, Dichgans, \& Hufschmidt, 1977 ) in the frontoparallel plane induces the same effect: rod adjustment bypasses the upright in the same direction as that in which the disk is rotating. In the RFT, the error in estimating the vertical is not necessarily directly attributable to biased visual directions, since the same errors can be induced by a rotating disk, which, as a rule, presents no directional conflict with gravity. In fact, these visual disturbances are tantamount to an indirect disturbance of the gravity cues. It is already known that visual and labyrinthine information converge in the vestibular nuclei and that the same cell can respond equally to a visual or a labyrinthine stimulation (Daunton, Christensen, \& Thomsen, 1981; Daunton \& Thomsen, 1979; Dichgans \& Brandt, 1972; Waespe \& Henn, 1977). A large tilted visual frame (with an angular size of more than

This study was sponsored by the Centre National de la Recherche Scientifique (CNRS, UA 665), the Ministère de la Défense (DRET contract 86/047), and the Université des Sciences Sociales de Grenoble. Address correspondence to Christian Marendaz, Laboratoire de Psychologie Expérimentale, Université des Sciences Sociales de Grenoble, BP 47X, 38040 Grenoble Cédex, France. $\left.10^{\circ}\right)$ also induces the illusion that the body is tilted in the opposite direction (Goodenough, Nowak, Oltman, Cox, \& Sigman, 1982; Sigman, Goodenough, \& Flannagan, $1978,1979)$. This optostatic vection is comparable to the optokinetic vections described by Clément, Jacquin, and Berthoz (1985), Delorme and Martin (1986), and Dichgans and Brandt (1978). This illusion of motion rotates the subjective vertical in the opposite direction (toward the tilted frame or the rotating disk). Rod adjustment errors relative to the upright, therefore, do not appear to be due to the direct influence of the visual field. Instead, they are attributable indirectly to the effect of visual field on posture. The between-subjects differences noted in the RFT (field dependence-independence) can thus result essentially from differences in optostatic vection sensitivity. Subjects who give preference to visual references in visual-postural conflict (field dependent) (see Goodenough, Oltman, \& Cox, 1987; Witkin, 1949; Witkin et al., 1954) are more sensitive to the effects of the direction given by the frame than are subjects who favor posturo-gravity references (field independent). These differences can be due to acquired postural equilibrium habits (Ohlmann, 1985, 1987).

Optostatic vection and optokinetic vection are produced due to specific properties of cells of the vestibular nuclei. It can be assumed that optostatic vection results from automatic subcortical mechanisms and, as such, is not controlled by the subject. Moreover, this roll visual stimula- 
tion can trigger extremely rapid postural readjustments and corollary modifications in the subjective vertical (Clément et al., 1985; Mauritz et al., 1977). Thus, if errors in the RFT are essentially an indication of the degree of sensitivity to vection produced by the frame, the automatic immediate nature of this sensitivity can lead one to expect a stable typology for the tachistoscopic RFT. In other words, what can be expected is a positive correlation between conditions in which the RFT is presented and solved in unlimited time and the tachistoscopic version in which display time is $130 \mathrm{msec}$ and solution time is about $750 \mathrm{msec}$. The fact that a momentarily tilted frame also can induce a stable rotation of space coordinates corroborates this hypothesis. Di Lorenzo and Rock (1982) asked their observers to put a frame initially tilted at $54^{\circ}$ into an upright position. Large adjustment errors $\left(7^{\circ}\right)$ were made in the same direction as that in which the frame initially was tilted. This large error cannot be explained simply by the effect of starting position. The adjustment of the rod never exceeds $1^{\circ}$ without any link with its starting position. The induction of spatial-postural modifications by a rotating disk or by a frame, even if it is not continuously present, suggests that subjective spatial axes are strongly disturbed in the early stages of solving the RFT task.

Furthermore, the effects of vections seem to be linked to the importance of peripheral visual stimulations, be it the retinal periphery or the in-depth periphery (Delorme \& Martin, 1986; Dichgans \& Brandt, 1978). Similarly, it has been observed that the angular size of the frame is of great importance in conditions such as in the RFT; the extent of the disturbance bears a positive correlation with this angular size (Ebenholtz, 1977; Ebenholtz \& Benzschawel, 1977; Ebenholtz \& Glaser, 1982; Streibel \& Ebenholtz, 1982). If this sensitivity to optostatic vection plays a great part in the disturbing effects of a tilted frame, one can therefore expect to find an effect involving angular size under tachistoscopic conditions, an effect that is similar to the one observed when display time is not limited.

Thus, the aim of this investigation was to study, in a general and differential perspective, using angular sizes of frames greater than $10^{\circ},(1)$ the extent of individual homogeneity of performances (correlations) between the traditional RFT (Oltman, 1968) and experimental conditions in which the RFT conflict is presented tachistoscopically, and (2) the effect of changing the angular sizes of the frame under only tachistoscopic conditions. Three operational hypotheses were tested: (1) Whether performances in the portable and tachistoscopic RFT conditions would correlate positively; (2) whether under tachistoscopic conditions, the mean deviation relative to the gravitational upright would increase as the angular size of the frame becomes larger; and (3) whether there would be an interaction between the angular size of frame and the degree of dependence. Subjects more prone to relying on the frame's spatial information (those tending toward field dependence) were expected to continue to be dependent even more so because of the frame's striking disturbing effect (see Hypothesis 2; Streibel \& Ebenholtz, 1982).

\section{METHOD}

\section{Subjects}

The experimental population consisted of 36 volunteer first-year students of psychology (28 males, 8 females).

\section{Apparatus}

The traditional portable RFT. The portable RFT (Oltman, 1968) is equipped with a response device that allows the subject to alter the rod's orientation. The angular size of the frame is $28^{\circ}$.

Tachistoscopic RFT. The magnitude of the angular size of the stimuli $\left(28^{\circ}\right.$ and $\left.50^{\circ}\right)$ and the necessity to avoid all contextual spatial references called for the setting up of a special experimental device. The stimuli were projected onto a circular screen $(150 \mathrm{~cm}$ in diameter), at the back of which was situated a black concentric cone $(60 \mathrm{~cm}$ deep) that opened into a space $30 \mathrm{~cm}$ in diameter. In the middle of this opening, a notch enabled the subject's head to be held in the orientation desired and in a sufficiently protruding position so as to avoid any "inhibition of contour," as might be the case because of the cone's circular nature (see Ebenholtz \& Utrie, 1982,1983 ). The stimuli were picture slides of computer-constructed figures. Stimuli with an angular size of $28^{\circ}$ were, from a physical standpoint, faithful reproductions of Oltman's (1968) portable RFT stimuli. When the angular size changes, the stimuli preserve a strict homothetia. The picture slides were projected from two machines equipped with electromagnetic shutters attached to an electronic control box. The display of the stimuli and the compilation of data were controlled by an Apple II microcomputer.

\section{Design}

The between-subjects factor was visual field dependence: dependent (FD) versus independent (FI). Both modalities were determined according to the break in the scores distribution near the median. The repeated factor was type of RFT condition: portable RFT versus tachistoscopic RFT at $28^{\circ}$ versus tachistoscopic RFT at $50^{\circ}$. The dependent variable was the average deviation from the upright, calculated using the unsigned errors in the RFT and the signed ones. ${ }^{2}$

\section{Procedure}

The experiment. The presentation of the portable RFT was carried out in the usual way (Oltman, 1968). However, only the four conditions at which the frame is tilted $28^{\circ}$ on the left were presented to the subjects. ${ }^{3}$ In the RFT tachistoscopic condition, for each angular size of the stimuli, each subject attempted a series of 25 trials repeated four times. During these trials, the rod's orientation changed and its contextual square frame remained tilted at $28^{\circ}$ on the left. The rod's orientation went from $17^{\circ}$ on the left to $7^{\circ}$ on the right with step-by-step changes of $1^{\circ}$ per change. The stimuli were presented using the constant method.

Testing. The traditional portable RFT was followed about 2 weeks later by tachistoscopic conditions of $28^{\circ}$ and $50^{\circ}$. The tachistoscopic conditions were subdivided into two experimental sessions according to the angular size of the stimuli. Each session lasted for about $15 \mathrm{~min}$, with a $1 / 2-\mathrm{h}$ intersession interval. The order of presentation of sessions was counterbalanced over the intersubjects FD versus FI factor.

Presentation of stimuli and instructions. Presentation of the stimuli on the tachistoscope was carried out in the following sequence: 800 msec after a starting signal that informed the subject that he/she was to gaze at the central dot, the stimulus was displayed for $130 \mathrm{msec}$ in the subject's frontoparallel plane. The stimulus was followed immediately by a mask. For each presentation, 
the subject was to reply as quickly and as accurately as possible whether or not the rod was upright. To do this, he/she pushed the touch button in his/her left hand if the rod was not upright and the one in his/her right hand if it was. The instructions emphasized the necessity of an accurate reply at the expense of rapidity.

\section{RESULTS $^{4}$}

To facilitate the presentation of the results, the experimental conditions-portable RFT, tachistoscopic RFT with $28^{\circ}$ square frame, and tachistoscopic RFT with $50^{\circ}$ square frame-are labeled, respectively, RFT.P.28, RFT.T.28, and RFT.T.50.

With the tachistoscopic situation, the signed and unsigned deviations from the upright were calculated by finding the average of the rod orientation values that corresponded to conditions in which the subject judged the rod as upright in $75 \%$ of the cases. This criterion of $75 \%$, corresponding in reality to three congruent responses out of four, is the minimum required to avoid any haphazard responses. In fact, an overall look at the results from the tachistoscopic conditions shows a certain coherence in the responses: whatever the level of the subjects' performances, haphazard answers were not very numerous, and the rod orientation values for which the subject answered in the affirmative were very close.

Table 1 sums up the indices of distribution concerning the mean individual deviations from the upright based on the various RFT conditions for the population as a whole and for each FD and FI group.

\section{Examination of the Individual \\ Intertask Homogeneity}

Hypothesis 1 suggested that relative homogeneity would occur in the same subject's performances under the different RFT conditions. This hypothesis is not weakened by the Kendall coefficient of concordance found with the three RFTs (P.28, T.28, T.50): $W_{\mathrm{s}}=.80, \chi^{2}=84$, $p<.001 ; w_{\mathrm{u}}=.83, \chi^{2}=87.15, p<.001$. A Spearman $\varrho$ carried out on the different conditions taken pair by pair reveals some variability in the correlation coefficient ${ }^{5}$ since the intrasubject homogeneity is at its highest under tachistoscopic conditions. The analysis of variance (ANOVA) carried out on the factors (type of RFT and FI/FD) shows that they have separate effects [FI/FD: $F_{\mathrm{s}}(1,34)=64.15, p<.001 ; F_{\mathrm{u}}(1,34)=68.78$, $p<.001$; type of RFT: $F_{\mathrm{s}}(2,68)=3.04, p=.054$ $\left.F_{\mathrm{u}}(2,68)=3.06, p=.053\right]$ and that there is an interaction $\left[F_{\mathrm{s}}(2,68)=7.56, p=.0011 ; F_{\mathrm{u}}(2,68)=9.49\right.$, $p<.001]$. With the intersubjects FI/FD factor, the variance analysis, on the whole, falls in line with Hypothesis 1 . The intergroup differences are maintained in the tachistoscopic conditions, a fact that fits in with the homogeneity across conditions, as reported in the previous analysis. However, the presence of an interaction between the RFT factor and FI/FD factor leads us to believe that individual homogeneity across conditions is modified by intergroup differences. The correlational intragroup analysis (Kendall coefficient of concordance calculated for each modality of the FI/FD factor) verifies this point. The null hypothesis is rejected with a probability varying from $p<.01$ to $p \leq .10$ [RFT (P.28, T.28, T.50): FI, $W_{\mathrm{s}}=.59, N=17, \chi^{2}=28, p<.05$; $W_{\mathrm{u}}=.71, N=16, \chi^{2}=32, p<.01 ;$ for FD, $W_{\mathrm{s}}=$ $.54, N=19, \chi^{2}=29, p<.05 ; W_{\mathrm{u}}=.48, N=20$, $\left.\chi^{2}=27.4, p \leq .10\right]$. By using Spearman's e correlations between the devices taken two by two, these coefficients of concordance can be decomposed (see Table 2).

The correlational analysis carried out on the basis of the FI/FD factor restricts the isomorphism of the individual positions observed throughout the experimental population across the different RFT conditions, and sustains the corroboration of Hypothesis 1 which ensues from the general correlational analysis. In fact, in both FD and FI subjects, there is no link between the portable RFT and both tachistoscopic RFTs. This absence of correlation cannot be attributed to the lack of fidelity of the tachistoscopic devices since they are correlated. On the other hand, the fidelity of the portable RFT has been well established. The principal difference between the portable and the tachistoscopic RFT conditions lies in the ex-

Table 1

Unsigned and Signed Means and Standard Deviations from the Upright (in Degrees) for All Subjects and in Relation to the Perceptual Style

\begin{tabular}{|c|c|c|c|c|c|c|c|c|c|}
\hline \multirow{2}{*}{$\begin{array}{l}\text { Perceptual } \\
\text { Style }\end{array}$} & \multicolumn{3}{|c|}{ RFT.P.28 } & \multicolumn{3}{|c|}{ RFT.T. 28} & \multicolumn{3}{|c|}{ RFT.T.50 } \\
\hline & $M$ & $S D$ & $N$ & $M$ & $S D$ & $N$ & $M$ & $S D$ & $N$ \\
\hline \multicolumn{10}{|c|}{ Signed } \\
\hline Total (FI + FD) & 4.50 & 4.17 & 36 & 3.39 & 2.40 & 36 & 4.28 & 3.33 & 36 \\
\hline FI & 1.22 & 1.01 & 17 & 1.93 & 1.87 & 17 & 2.13 & 1.59 & 17 \\
\hline FD & 7.78 & 3.39 & 19 & 4.85 & 1.95 & 19 & 6.40 & 3.20 & 19 \\
\hline \multicolumn{10}{|c|}{ Unsigned } \\
\hline Total (FI+FD) & 5.18 & 4.05 & 36 & 3.87 & 2.01 & 36 & 4.67 & 3.01 & 36 \\
\hline FI & 1.63 & 0.63 & 16 & 2.46 & 1.35 & 16 & 2.52 & 1.03 & 16 \\
\hline FD & 8.02 & 3.30 & 20 & 5.00 & 1.74 & 20 & 6.39 & 2.99 & 20 \\
\hline
\end{tabular}

Note-RFT.P. 28 = portable rod-and-frame test (RFT) with $28^{\circ}$ square frame. RFT.T. $28=$ tachistoscopic RFT with $28^{\circ}$ square frame. RFT.T.50 = tachistoscopic RFT with $50^{\circ}$ square frame. FI = field-independent perceptual style. $F D=$ field-dependent perceptual style. 
Table 2

Correlations (Spearman's rho) Among the Three RFT

Conditions (Unsigned and Signed Data) for All Subjects and in Relation to the Perceptual Style

\begin{tabular}{|c|c|c|c|c|c|c|c|c|c|}
\hline \multirow{3}{*}{$\begin{array}{l}\text { Field-Dependent } \\
\text { Style } \\
\end{array}$} & \multicolumn{9}{|c|}{ Field-Independent Style } \\
\hline & \multicolumn{3}{|c|}{ RFT.P.28 } & \multicolumn{3}{|c|}{ RFT.T.28 } & \multicolumn{3}{|c|}{ RFT.T.50 } \\
\hline & e & $p$ & $N$ & e & $p$ & $N$ & e & $p$ & $N$ \\
\hline \multicolumn{10}{|c|}{ Signed } \\
\hline RFT.P. 28 & & & & .06 & $>.20$ & 17 & .16 & $>.20$ & 17 \\
\hline RFT.T. 28 & .20 & $>.20$ & 19 & & & & .82 & $<.001$ & 17 \\
\hline RFT.T.50 & .20 & $<.20$ & 19 & .46 & $=.047$ & 19 & & & \\
\hline \multicolumn{10}{|c|}{ Unsigned } \\
\hline RFT.P.28 & & & & 42 & $=.10$ & 16 & .37 & $=.16$ & 16 \\
\hline RFT.T.28 & .00 & $>.20$ & 20 & & & & .92 & $<.001$ & 16 \\
\hline RFT.T.50 & .13 & $>.20$ & 20 & .54 & $=.014$ & 20 & & & \\
\hline
\end{tabular}

Note-RFT.P. 28 = portable rod-and-frame test (RFT) with $28^{\circ}$ square frame. RFT.T.28 = tachistoscopic RFT with $28^{\circ}$ square frame. RFT.T.50 = tachistoscopic RFT with $50^{\circ}$ square frame.

posure time of the stimulus. The effect was more strictly tested by comparing performances carried out under conditions in which the display time varied while the angular size remained constant (RFT.P.28 and RFT.T.28). The ANOVA between type of RFT (P.28 vs. T.28) and perceptual style (FI vs. FD) shows that RFT.P.28 and RFT.T.28 differ significantly $\left[F_{s}(1,34=4.63, p=.039\right.$; $\left.F_{\mathrm{u}}(1,34)=4.88, p=.034\right]$, and that the factors (type of RFT and perceptual style) interact symmetrically $\left[F_{\mathrm{s}}(1,34)\right.$ $\left.=12.46, p=.0012 ; F_{\mathrm{u}}(1,34)=15.15, p<.001\right]$ (see Table 1). In the RFT.T.28 condition, an improvement in the FD subjects' performances was observed; for subjects tending toward FI, a deterioration was observed. However, the analytical intragroup comparisons (simple effects) indicate that this variation of results is significant only in the case of subjects tending toward FD $\left[F_{\mathrm{s}}(1,34)\right.$ $\left.=17.1, p<.001 ; F_{\mathrm{u}}(1,34)=20.99, p<.001\right]$. In accepting that the essential factor in differentiating the RFT.P.28 and RFT.T.28 is time exposure (unlimited vs. limited to $130 \mathrm{msec}){ }^{6}$ we think that this form of interaction might be interesting for the comprehension of the processes involved in solving the RFT conflict.

The fact that the correlation between the portable and tachistoscopic conditions observed in the overall population disappears in each perceptual style, added to the fact that FD subjects perform better under tachistoscopic conditions, permits the assumption that a secondary visual process is used in the portable RFT. This process is independent of the automatic process common to all RFT conditions (optostatic vections) and has a temporal feature. It most likely involves focal vision relative to the visual-postural relationship between rod and frame. This process is termed "secondary"; although it can modify the intrastyle hierarchy and the averages in each group, it does not appear to be effective enough to reorganize the interindividual hierarchy as a whole (see above correlational analysis of the overall experimental population). One might say that this process has to do with the possi- bility of a foveal exploration of the contextual frame; in other words, it is linked to ocular movements. Insofar as FI subjects favor postural references (for reasons still to be explained) in order to solve the RFT, the display time affects their performance only slightly, except when too short an exposure time lessens the precision of capturing information about the target. The same does not hold in the case of FD subjects, who supposedly opt for visual information in a visual-postural conflict. It can be assumed that the FD subjects' primary sensitivity to contextual visual references (experimental condition permitting) leads them to carry out a foveal exploration of the disturbing frame. The drop in their performance is thus an indication of their usage of this extra information. It must be noted that the testing of this hypothesis began with the study of the subjects' eye strategies in the portable RFT. Results show that ocular movements are not haphazard, but are linked with FI and FD styles: the percentages of target fixations vary from $7 \%$ in most FI subjects to $20 \%$ in most FD subjects (Cian \& Ohlmann, 1987).

\section{Interaction Between the Angular Size of the Frame in Tachistoscopic RFT and FI/FD}

To test Hypotheses 2 and 3, which are based on the role of the change in angular sizes under tachistoscopic conditions, notably in interaction with the FI/FD factor, we carried out an ANOVA based on the factors angular size of the tachistoscopic RFT frames $\left(28^{\circ}\right.$ vs. $\left.50^{\circ}\right)$ and perceptual style (FI vs. FD). The resuit is that, apart from the FI/FD effect $\left[F_{\mathrm{s}}(1,34)=30.74, p<.001 ; F_{\mathrm{u}}(1,34)\right.$ $=32.68, p<.001]$, as expected in Hypothesis 2 , the mean error increases as the angular size gets larger $\left[F_{\mathrm{s}}(1,34)=4.98, p=.03 ; F_{\mathrm{u}}(1,34)=3.93, p=.056\right]$. However, interaction between both factors in Hypothesis 3 is significant only at $p<.10\left[F_{\mathrm{s}}(1,34)=3.01, p\right.$ $\left.=.09 ; F_{\mathrm{u}}(1,34)=3.3, p=.078\right]$. Nevertheless, because the angular size factor has a similar influence on the raw data of FD and FI subjects, the statistical emergence of such an interaction is difficult. In fact, the analytical intragroup comparisons (simple effects) indicate that subjects who tend toward FD commit more errors as the angular size of the frame increases $\left[F_{\mathrm{s}}(1,34)=8.32, p=\right.$ $\left..007 ; F_{\mathrm{u}}(1,34)=8.11, p<.007\right]$, whereas those who tend toward FI are shown statistically to be unaffected by this factor $[F(1,34)<1]$. This pattern of results is similar to that obtained by Streibel and Ebenholtz (1982). In their experiment, the display time was not limited and the angular size variation across conditions was larger, with a size ratio of $1: 4.5$ as opposed to one of $1: 78$ in the present experiment.

\section{DISCUSSION}

The aim of this experiment was to point out that, in solving vision-posture conflicts such as those found in the RFT, a tachistoscopic presentation of stimuli would hardly affect the perceptual behavior of adult subjects, that is, 
their ability to turn a rod upright within a disturbing visual field context. The general analysis does not invalidate this hypothesis: it has been observed, from tachistoscopic presentations, that the angular size of frame factor has an effect similar to that described in the literature on unlimited time presentation and that there is a ranking of subjects that is statistically very close regardless of the exposure time.

These results (obtained by using an exposure time of $130 \mathrm{msec}$ and a mean response time of about $750 \mathrm{msec}$ ) are compatible with the idea that the RFT can be solved mainly by neurosensory (subcortical) processes (Ebenholtz, 1985a, 1985b); moreover, it can be assumed that these noncognitive and automatic processes are comparable to those involved in optic vections. However, these results do not fall in line with any account for the RFT error, which might be based on normalization mechanisms (in terms of a time adaptation phenomenon) as was proposed fairly explicitly by Di Lorenzo and Rock (1982) and Rock (1983) in their righting effect theory. According to these authors, when the angular size of the frame is sufficient $\left(>10^{\circ}\right)$, the RFT error results from an underestimation of the frame's tilt owing to "the tendency on the part of the visual system to accept a tilted frame of reference as upright or as less tilted phenomenally than it is objectively" (Di Lorenzo \& Rock, 1982, p. 537). Even if the processing nature of this tendency (overconstancy of initial reaction vs. normalization) has not been precisely dealt with in this theoretical formulation, on an operational level, a normalization procedure was used for testing this hypothesis: each time, before returning the frame (alone) to the upright position (the rod too but in a tilted frame), the subject was shown the tilted frame for a period of $4 \mathrm{~min}$. The close link between performances carried out within a limited or an unlimited time casts doubt on any explanation based on time adaptation as to why there is a mistaken judgment of the upright.

The stability in the intersubject differences throughout all the devices emphasizes the importance of an automatic, primary process, whereas the absence of any intrastyle correlation and the improvement in the FD subjects' performances under tachistoscopic conditions point to the existence of a more cognitive secondary process. This secondary process is limited to the portable RFT, which denotes the former's temporal characteristic. This process might involve the foveal exploration of visual references in controlling the orientation of the rod. With a tachistoscopic presentation, the impossibility of carrying out eye movements curbs FD subjects' usage of the visual references from the frame and, as such, allows them to perform better.

Thus, at least two distinct processes intervene in solving the RFT. The first, vections, has to do with the subjects' postural orientation and plays a large role in organizing intersubject differences. The second process, a more cognitive one with a less striking effect, involves the orientation of the rod relative to the visual and postural orientations available. It possibly has more to do with selec- tion of reference frames in perceptual organization (see Humphreys, 1983; Marendaz, 1987; Palmer, 1983). The effects of these independent processes may be combined additively.

\section{REFERENCES}

Cian, C., \& Ohlmann, T. (1987, September). Differential study of eye movements during optostatic vection induced by a tilted frame. Paper presented at The Fourth European Conference on Eye Movements, Göttingen, Federal Republic of Germany.

Clément, G., Jacquin, I., Berthoz, A. (1985). Habituation of postural readjustment induced by motion of visual scenes. In $\mathbf{M}$. Igarashi \& F. O. Black (Eds.), Vestibular and visual control on posture and locomotor equilibrium (pp. 99-104). Basel, Switzerland: Karger. Daunton, N. G., Christensen, C.A., Thomsen, D. D. (1981). Visual modulation of otolith responses: A paradigm for the study of self-motion perception and its neural substrate. In T. Gualtierroti (Ed.), The vestibular system: Function and morphology (pp. 452-462). New York: Springer-Verlag.

Daunton, N., Thomsen, D. (1979). Visual modulation of otolithdependent units in cat vestibular nuclei. Experimental Brain Research, 37, 173-176.

Delorme, A., MARTIN, C. (1986). Role of retinal periphery and depth periphery in linear vection and visual control of standing in humans. Canadian Joumal of Psychology, 40, 176-187.

DichGans, J., \& BRANDT, T. (1972). Visual-vestibular interaction and motion perception. In J. Dichgans \& E. Bizzi (Eds.), Cerebral control of eye movements and motion perception (pp. 327-338). Basel, Switzerland: Karger.

Dichgans, J., \& BRANDT, T. (1978). Visual-vestibular interaction: Effects on self-motion perception and postural control. In R. Held, H. W. Leibowitz, \& H.-L. Teuber (Eds.), Handbook of sensory physiology: Vol. 7. Perception (pp. 756-804). New York: Springer-Verlag.

Dichgans, J., Young, L. R., Brandt, T., Held, R. (1972). Moving visual scenes influence apparent direction of gravity. Science, 178, 1215-1217.

D1 LoREnzo, J. R., Rock, 1. (1982). The rod and frame effect as a function of righting the frame. Journal of Experimental Psychology, 8, 536-546.

EBENHOLTZ, S. M. (1977). Determinants of the rod and frame effect: The role of retinal size. Perception \& Psychophysics, 22, 531-538.

Ebenholtz, S. M. (1985a). Absence of relational determination in the rod-and-frame effect. Perception \& Psychophysics, 37, 303-306.

EBEnholtz, S. M. (1985b). Depth separation fails to modulate the orientation-inhibition effect. Perception \& Psychophysics, 37, 533-535.

Ebenholtz, S. M., \& Benzschawel, T. L. (1977). The rod and frame effect and induced head tilt as a function of observation distance. Perception \& Psychophysics, 22, 491-496.

Ebenholtz, S. M., Glaser, G. W. (1982). Absence of depth processing in the large-frame rod-and-frame effect. Perception \& Psychophysics, 32, 134-140.

Egenholtz, S. M., \& UTrue, J. W., JR. (1982). Inhibition of the rodand-frame effect by circular contours. Perception \& Psychophysics, 32, 199-200.

Ebenholtz, S. M., Utrie, J. W., JR. (1983). Peripheral circular contours inhibit the visual orientation control system. Aviation, Space \& Environmental Medicine, 54, 343-346.

Goodenough, D. R., Nowak, A., Oltman, P. K., Cox, P. W., \& SIGMan, E. (1982). A visually induced illusion of body tilt in a horizontal plane. Perception \& Psychophysics, 31, 419-424.

Goodenough, D. R., Oltman, P. K., Cox, P. W. (1987). The nature of individual differences in field dependence. Journal of Research in Personality, 21, 81-99.

Goodenough, D. R., Oltman, P. K., Sigman, E., Rosso, J., \& MerTZ, H. (1979). Orientation contrast effects in the rod-and-frame test. Perception \& Psychophysics, 25, 419-424.

Hughes, P. C., Brecher, G. A., Fishin, S. M. (1972). Effects of rotating backgrounds upon the perception of verticality. Perception \& Psychophysics, 11, 135-138. 
HUMPHREYs, G. W. (1983). Reference frames and shape perception. Cognitive Psychology, 15, 151-196.

Marendaz, C. (1987). Contextes, référentiels et fonctionnements perceptifs. In M. Reuchlin, F. Longeot, C. Marendaz, \& T. Ohlmann (Eds.), Connaitre différemment (pp. 43-64). Nancy, France: Presses Universitaires de Nancy.

Mauritz, K. H., Dichgans, J., \& Hufschmidt, A. (1977). The angle of visual roll motion determines displacement of subjective vertical. Perception \& Psychophysics, 22, 557-562.

OHLManN, T. (1985). Variabilité intraindividuelle et fonctionnement cognitif. In J. Drévillon, M. Huteau, F. Longeot, M. Moscato, \& T. Ohlmann (Eds.), Fonctionnement cognitif et individualité (pp. 185230). Brussels, Belgium: Mardaga.

OhlmanN, T. (1987). La perception de la verticale lors de conflits vision/posture: Un exemple de processus vicariants. In M. Reuchlin, F. Longeot, C. Marendaz, \& T. Ohlmann (Eds.), Connaître différemment (pp. 19-42). Nancy, France: Presses Universitaires de Nancy.

Oltman, P. K. (1968). A portable rod-and-frame apparatus. Perceptual \& Motor Skills, 26, 503-506.

OTOOLE, B. I. (1979). Exposure-time and spatial-frequency effects in the tilt illusion. Perception, 8, 557-564.

Palmer, S. E. (1983). The psychology of perceptual organization: A transformal approach. In J. Beck, B. Hope, \& A. Rosenfeld (Eds.), Human and machine vision (pp. 269-339). New York: Academic Press.

Rock, I. (1983). The logic of perception. Cambridge, MA: Bradford Books/MIT Press.

SEKUler, R., \& LitTLEJOHN, J. (1974). Tilt aftereffect following very brief exposures. Vision Research, 14, 151-152.

Sigman, E., Goodenough, D. R., Flannagan, M. (1978). Subjective estimates of body tilt and the rod and frame test. Perceptual \& Motor Skills, 47, 1051-1056.

Sigman, E., Goodenough, D. R., \& Flannagan, M. (1979). Instructions, illusory self tilt and the rod and frame test. Quarterly Journal of Experimental Psychology, 31, 155-165.

Streibel, M. J., \& Ebenholtz, S. M. (1982). Construct vaidity of perceptual style: Role of stimulus size in the embedded-figures test and the rod-and-frame test. Perception \& Psychophysics, 31, 128-138.

WAESPE, W., \& HeNN, V. (1977). Neuronal activity in the vestibular nuclei of the alert monkey during vestibular and opto-kinetic stimulation. Experimental Brain Research, 27, 523-538.

WitkIN, H. A. (1949). Perception of body position and of the position of the visual field. Psychological Monographs, 63, 1-46.

WITKIN, H. A., \& AsCH, S. (1948). Studies in space orientation: IV. Perception of the upright in the absence of visual field. Further experiments on perception of the upright with visual fields. Journal of Experimental Psychology, 38, 762-782.

Witkin, H. A., Lewis, H. B., Hertzman, M., Machover, K., WeissNER, B. P., \& WAPNER, S. (1954). Personality through perception. New York: Harper.

\section{NOTES}

1. Another fact, tilt contrast, is tachistoscopically resistant ( $O$ 'Toole, 1979; Sekuler \& Littlejohn, 1974). On the one hand, this presumed neurosensorial process yields approximately only $1^{\circ}$ of error in this task (Goodenough, Oltman, \& Cox, 1987; Goodenough, Oltman, Sigman, Rosso, \& Mertz, 1979; Rock, 1983). On the other hand, the righting of a frame alone gives a similar result as the righting of the rod in the RFT (Di Lorenzo \& Rock, 1982); this correlation cannot be explained here by the tilt contrast absent in the former condition.

2. When the rod-and-frame effect (RFE) is based on signed errors, the deviations from the true gravitational upright in the direction opposite that of the frame tilt are coded as negative values. When the RFE is based on unsigned errors, the deviations from the true gravitational upright are coded without taking the direction of errors into account. This second method of calculation is proposed here because it corresponds to the overall visual field limited to a frame, according to Witkin and Asch's (1948) original definiton. Thus, when a subject carries out an absolute deviation greater than his/her algebraic deviation, he/she can be said to have undergone the phenomenon known as perceptual shift. The references used, therefore, are the sides tilted at $62^{\circ}$ instead of those tilted at $28^{\circ}$. The alphanumerical symbols used in the statistical calculations are labeled $s$ (signed) and $u$ (unsigned) in order to indicate the dependent variable used in calculating.

3 . In order not to prolong the testing time, only one frame orientation ( $28^{\circ}$ on the left, counterclockwise) has been exposed. This tilt usually causes more striking deviations from the upright than does the one $28^{\circ}$ on the right (clockwise).

4. Since the instructions were essentially based on the accuracy of the response, the latency of the responses was measured (in milliseconds), for information only, under tachistoscopic situations. The correlational and variance analyses based on the response time (because the instruction called for accuracy rather than speed) show that the response time (RT) varied depending on the angular size of the frame but not according to the FI/FD factor: the RT was smaller when there was no disturbing frame factor $[715$ vs. $757 \mathrm{msec} ; F(1,34)=9.93, p=.003]$ or when the angular size was smallest [696 vs. $776 \mathrm{msec} ; F(1,34)=17.9$, $p<.001]$. There was a steady individual homogeneity across conditions for the RT $(r=.72, p<.001)$, but there was no relationship between the speed and accuracy of the response $(r=.05)$.

5. The correlations between the various RFT conditions are as follows: (1) signed scores: RFT.P. $28 \times$ RFT.T.28, $\mathrm{Q}=.60 ;$ RFT.P. 28 $\times$ RFT.T. $50, e=.65 ;$ RFT.T. $28 \times$ RFT.T.50, $\varrho=.81$; (2) unsigned scores: RFT.P. $28 \times$ RFT.T. $28, e=.63$; RFT.P. $28 \times$ RFT.T.50, $\varrho$ $=.69 ;$ RFT.T. $28 \times$ RFT.T.50, $e=.85$.

6. Some differences exist between both conditions: self-adjustment in the portable RFT, tridimensionality of the disturbing tunnel, and coplanarity of the frame and rod. However, it has been systematically shown that these factors do not influence the quality of the performance (Ebenholtz \& Glaser, 1982; Oltman, 1968; Witkin, 1949; Witkin \& Asch, 1948; Witkin et al., 1954). 